\title{
Focused attention in three-dimensional space
}

\author{
GEORGE J. ANDERSEN \\ University of Illinois, Urbana-Champaign, Illinois
}

\begin{abstract}
The size of focused attention was assessed within a three-dimensional display. Subjects viewed random-dot stereogram displays in which they responded differentially to vertical and horizontal bars. Adjacent noise elements either were identical to the response target or specified the opposite response. The position of the noise elements was varied in depth according to binocular disparity. Interference by incompatible noise elements decreased with depth separation between the noise elements and response target. In addition, interference was greater for noise elements that were more distant from the observer than from the response target than it was for noise elements that were closer to the observer than to the response target. The implications of these results for a viewer-centered representation of focused attention in depth are discussed.
\end{abstract}

An important property of visual processing is the ability to allocate processing resources or attend to locations in the visual field that might contain important information. Considerable research has been conducted to determine the spatial limits of visual attention when subjects are required to attend to information at a specified position in the visual field. B. A. Eriksen and C. W. Eriksen (1974) presented subjects with five simultaneous items in visual displays. The subjects were required to respond to the middle item of each display and to ignore the adjacent noise elements that were present. The response specified by the adjacent set of elements was either compatible or incompatible with the response to the central target. By varying the spatial separation of the noise elements relative to the central target, the size of focused attention could be measured. If the noise elements fell within the focus of attention, reaction time (RT) would be greater when they were incompatible with the response to the central target than when they were compatible.

Using this paradigm, B. A. Eriksen and C. W. Eriksen (1974) found that the interfering effects of the incompatible noise elements decreased with greater spatial separations between the target and noise elements up to $1^{\circ}$. In other studies containing similar paradigms, similar limits have also been found (C. W. Eriksen \& Hoffman, 1973; Hoffman \& Nelson, 1981; Posner, Nissen, \& Ogden, 1978). (For a review of the research on spatial attention, see Duncan, 1984.)

However, other studies have yielded evidence that the spatial limits of attention are greater than the $1^{\circ}$ limit. LaBerge (1983) presented subjects with displays containing letters that sometimes formed words. Some subjects were required to attend to single letters, whereas other

This research was supported by NSF Grant BNS 8607212. The author would like to thank C. Eriksen, A. Kramer, L. Krueger, G. Logan, R. Proctor, and two anonymous reviewers for comments on an earlier draft of the manuscript, and A. Needham for running the subjects. Reprint requests can be sent to George J. Andersen, Department of Psychology, University of Illinois, 603 E. Daniel St., Champaign, IL 61820. subjects were required to attend to entire words. The size of the focus of attention, as measured with a response target that varied in horizontal position, was larger for the subjects who were required to attend to words. LaBerge proposed that attention operated like a spotlight in the visual field. Items falling within the beam of this spotlight received processing priority over items not falling within the "beam" of attention.

More recently, the spotlight theory was modified to include the possibility that the focus of attention might vary much like the focus of a zoom lens (C. W. Eriksen \& St. James, 1986; C. W. Eriksen \& Yeh, 1985). According to this view, the size and strength of perceptual processing might vary in accordance with the available information within the visual field. If necessary, attention could be distributed over the entire visual field, but it would have limited strength in any given region, because processing capacity would be spread across the visual field. On the other hand, the size of attention could be reduced to a small area of the visual field, which would permit the concentration of processing capacity on a small region of the visual field.

Another variant of this approach is that the allocation of processing priority might vary according to the position of the items within the focus of attention (LaBerge \& Brown, 1986). According to this view, targets that fall within the central regions of the spotlight would receive the greatest priority for processing, whereas items that are located farther away from this central position, but still fall within the spotlight of attention, would receive less priority for perceptual processing. Thus, the allocation of attention can be viewed as a gradient of processing (LaBerge \& Brown, 1989).

In general, research on the size of focused attention has involved the investigation of processing limitations when an item at a specific location is attended to in a twodimensional (2-D) display. No research has been designed to investigate the size of focused attention within a threedimensional (3-D) scene. There have, however, been two studies in which the movement of attention (shifting the 
focus of attention from one location to another location) in a 3-D scene was investigated. Downing and Pinker (1985) required subjects to attend to the central position within an array of lights in a 3-D scene. The lights were organized along different visual directions in two rows located at different distances from the subject. A cue presented at the central location indicated the visual direction in which a light might appear. Responses were slower for targets positioned farther away than for closer targets. In addition, the cost of attending to farther targets increased with increased retinal eccentricity. They proposed that the mental representation underlying visual attention was similar to the 21/2-D sketch proposed by Marr (1982; Marr \& Nishahara, 1978), in which depth and visual angle were important in the underlying representation.

Gawryszewski, Riggio, Rizzolatti, and Umiltà (1987) also investigated the movement of attention in depth. Subjects were presented with a central stimulus that cued the subject to a position along the same visual direction that was either closer or farther away than the central stimulus. A response target was then presented at either of these two positions. On some of the trials, the central cue was valid, but on other trials, the cue was invalid. Mean RTs were greater for invalid cues than for valid cues, suggesting that the subjects could not simultaneously attend to targets positioned at different distances.

In another type of research involving the effects of depth variations on attention, Nakayama and Silverman (1986) investigated the usefulness of depth information as a discriminating feature in a visual search task. The target to be identified was embedded within a field of noise items in a stereoscopic display. If the target was located at a different depth plane than the noise items were, then the search for the target proceeded in parallel. This occurred for a variety of combinations of perceptual information used to define the noise items.

Although the three studies discussed above examined the role of depth information on attention switching (Downing \& Pinker, 1985; Gawryszewski et al., 1987) and visual search (Nakayama \& Silverman, 1986), they did not assess the size of focused attention in 3-D space. The purpose in the present study was to determine the limitations of focused attention within a 3-D display. Two issues were considered important in determining the size and strength of focused attention within a 3-D display. First, the allocation of processing resources might vary according to distance along the depth axis from the attended position. More specifically, fewer processing resources might be allocated to an item with increased distance between the item and the attended position along the depth axis. This is consistent with the view that attention is a gradient of processing capacity (see LaBerge \& Brown, 1989) and could be described metaphorically as a limitation in depth of focus (the range of distances over which objects are simultaneously in focus).

Second, the size and strength of focused attention may depend on the type of underlying perceptual representa- tion. Two types of representations (viewer-centered; object-centered) have been proposed to be recoverable from visual information (Marr \& Nishihara, 1978). In a viewer-centered representation, the distances of feature points are described in relation to the viewer's position. If the allocation of processing resources occurs within a viewer-centered representation, then near/far relations, relative to the observer, should be important. This type of representation can be considered an extension of the spotlight metaphor of attention, in which the region of attended space might be described by a solid visual angle (i.e., a conical region) and would be consistent with the $21 / 2$-D sketch stage in the model of vision proposed by Marr (1982). For this type of model, an asymmetry in the allocation of resources should occur according to near/far relations, because the area of focused attention would be greater for far items than for near items. This suggests that the interference of extraneous visual information with the identification of a target might depend on whether the interfering information is positioned either closer to or farther away from the observer than the primary target.

In an object-centered representation, the distances of feature points are described relative to each other or to the object. In this type of representation, no information is available that specifies the distance of the viewer relative to the object (Braunstein, 1988). Thus, information regarding near/far relations is not preserved. This type of representation is comparable to the 3-D model stage of Marr's (1982) model. Although visual information located at different distances from an attended position might be allocated varying amounts of resources, there should be no asymmetry based on near/far relations, because the distances of elements relative to the viewer are not specified. Thus, the interference of extraneous visual information with the identification of a target should be the same, regardless of whether the interfering information is positioned closer to or farther away from the observer than the primary target.

The purpose in the present study was to determine the size of focused attention within a 3-D representation and to determine whether the representation was viewercentered or object-centered. Random-dot stereograms were used to present depth information to the subjects. There are several advantages to using this type of information for depth. First, the items were embedded within a field of noise. Thus, in order to detect the target, the subjects needed to fuse the display. Second, the only depth information present was binocular disparity. Downing and Pinker (1985) and Gawryszewski et al. (1987) presented the targets within a real 3-D scene. Thus, it is unclear what information might have been used to determine a perception of depth. Also, with a real 3-D scene, the obtained effects may have resulted from visual factors such as shifts in accommodation or eye convergence rather than attentional processing. The displays in the present study were viewed through a stereoscopic prism viewer. This 
viewing method presents a collimated image (focused at infinity) to the viewer and reduces the likelihood that subjects would shift their accommodative focus.

The displays contained a central target and adjacent noise elements that were compatible or incompatible with the response to the central target. The noise elements were positioned closer than, at the same distance as, or farther away than the central target, depending on their disparity relative to the central target. If RTs were greater for trials that contained incompatible noise elements than for those that contained compatible noise elements, and if the degree of interference depended on whether the noise elements were farther away or nearer than the central target, then this would be consistent with a viewer-centered representation. However, if the degree of interference for near versus far noise elements decreased as disparity difference increased, and if the decrease was symmetrical (i.e., the same for near and far noise elements), then this would be consistent with an object-centered representation.

\section{EXPERIMENT 1}

Each trial involved the following sequence of events: First, the subject saw a random-dot stereogram that displayed a cross located in front of a background plane (see Figure 1). Once the display was fused, the subject pressed a key to initiate the trial. The fusion display was used to ensure that the subject had the stereo image fused. It was replaced with a random-dot precue display that contained either a solid rectangle or a solid circle. The precue display was used to ensure that the subject was attending to the location where the response target would be presented. If the precue was a rectangle, then the subject was to respond to the central target present in the display that immediately followed. If the precue was a circle, then the subject was not to respond to the central target. The rectangle or circle display was replaced by a response display.
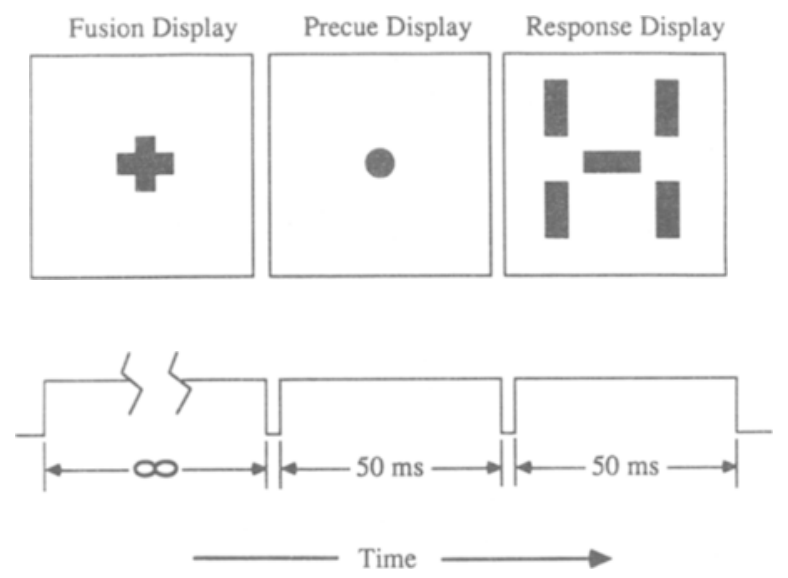

Figure 1. A schematic diagram of the sequence of displays presented on each trial. The interstimulus interval between displays was $3 \mathrm{msec}$.
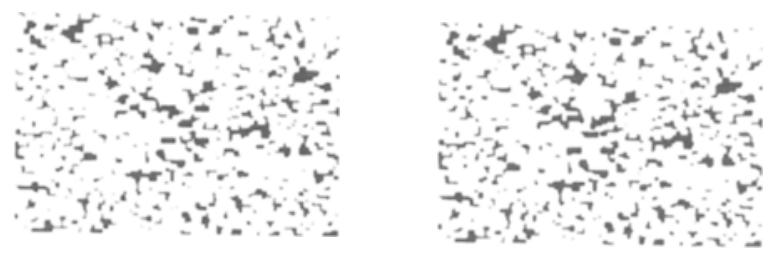

Figure 2. The response display used in Experiment 1.

The random-dot response display consisted of a central target, which determined which response was correct, and four adjacent noise elements (see Figure 2). The four noise elements surrounding the central target were either identical to the central target (for compatible noise conditions) or of the opposite target type (and thus incompatible with the correct response to the central target). The position of the noise elements as defined by disparity was closer than the central target (crossed disparity), at the same distance as the central target, or farther away than the central target (uncrossed disparity). Perceptually, the response display appeared as a randomly textured frontal parallel surface with a central target floating in front of the background surface. The adjacent elements also appeared to float in front of the background surface, with the depth position of the elements changing across trials according to the depth condition (disparity value) examined. Thus, for a crossed disparity trial, the noise elements appeared to be closer than the central target, whereas for an uncrossed disparity trial, the noise elements appeared to be farther away than the central target.

\section{Method}

Subjects. The subjects were 18 University of Illinois undergraduates who were paid for their participation. All subjects had normal or corrected-to-normal vision. The subjects were tested on a Randot stereotest. This test measures minimal detectable disparity by having subjects indicate which of three circles is closer for disparities ranging from $400^{\prime \prime}$ to $20^{\prime \prime}$ of arc. Data were excluded for two subjects; one failed to respond correctly on the circles test with a $70^{\prime \prime}$ of arc disparity value, and the other failed to show any sensitivity to binocular disparity information on the Randot test.

Design. Two independent variables were examined: type of noise element (compatible or incompatible), and the difference in disparity between the central target and noise elements $\left(-30^{\prime},-20^{\prime}\right.$, $-10^{\prime}, 0^{\prime}, 10^{\prime}, 20^{\prime}$, or $30^{\prime}$ of arc).

Stimuli. The displays were random-dot stereograms similar to those used by Julesz (1971). The random-dot stereograms were generated according to the following procedure: The background field was generated by randomly positioning 2,166 solid rectangles $\left(0.32^{\circ} \times 0.25^{\circ}\right)$ within the confines of a square region $\left(15.6^{\circ}\right.$ $\times 12.5^{\circ}$ ). The same randomly textured background field was presented to each of the subjects. To produce binocular disparity between the central targets and the background, the cross, precue, and response target were shifted to the right in the left field and to the left in the right field. Variations in the depth positions of the noise elements were produced by shifting the positions of the elements relative to the response target. Shifting the noise elements in the nasal direction produced crossed disparity relative to the response target; shifting the noise elements in the temporal direction produced uncrossed disparity relative to the response target. 
Three types of stereo displays were used on each trial: a fusion display, a precue display, and a response display. The fusion display consisted of a cross positioned in front of a randomly textured background field. This display was used to ensure that the subject had fused the stereo display before continuing with the trial. The dimensions of the cross were $0.32^{\circ} \times 0.73^{\circ}$ and $0.63^{\circ} \times 0.25^{\circ}$ for the vertical and horizontal bars that defined the cross. The disparity value of the cross was $40^{\prime}$ of arc relative to the background field.

The precue display consisted of either a circle or a rectangle positioned in front of the random background field. The dimensions of the rectangle were $0.63^{\circ} \times 0.73^{\circ}$. The radius of the circle was $0.63^{\circ}$. The disparity value of the precue targets, as well as the subsequent response target, was $40^{\prime}$ of arc relative to the background field.

The response display consisted of a central target (a horizontal or a vertical bar) surrounded by four noise elements. The noise elements were all vertical bars or all horizontal bars. The dimensions of the vertical and horizontal bars were $0.32^{\circ} \times 0.73^{\circ}$ and $0.63^{\circ}$ $\times 0.25^{\circ}$, respectively. The depths of the noise elements were $-30^{\prime}$, $-20^{\prime},-10^{\prime}, 0^{\prime}, 10^{\prime}, 20^{\prime}$, or $30^{\prime}$ of arc binocular disparity relative to the central stimulus. (Since disparity is measured relative to the position in depth on which the eyes are converged, which should be the position of the fusion, precue, and response targets in this experiment, the crossed and uncrossed disparities of the noise elements would be determined relative to the position of the central targets. Thus, the disparities of the noise elements represent values relative to the central target and not the background).

The four noise elements were presented (in the frontal parallel plane) around the central target at $45^{\circ}, 135^{\circ}, 215^{\circ}$, and $305^{\circ}$ positions. Thus, the noise elements were positioned at the corners of a square region, with the central target positioned at the center of the square (see Figure 2). The edge-to-edge separation (measured as the minimal corner-to-corner separation) between the noise elements and central target was $0.42^{\circ}$. Trials were either compatible (the central target and noise elements were identical) or incompatible (the central target and noise elements were different). The position of the noise elements was shifted in equal increments in both images to maintain a constant visual angle separation between the central target and noise elements.

The background random-dot field was the same for the fusion, precue, and response displays. In addition, the fusion, precue, and central target were always located at the same disparity value $\left(40^{\prime}\right.$ of arc) relative to the background and at the same vertical and horizontal position. The duration of the precue display was $50 \mathrm{msec}$. The duration of the response display was also $50 \mathrm{msec}$. The interstimulus interval (ISI) between the displays was $3 \mathrm{msec}$. Thus, the total duration of each trial was $103 \mathrm{msec}$, which was below the minimum time required to initiate an eye vergence shift (Rashbass \& Westheimer, 1961; Westheimer \& Mitchell, 1969).

Apparatus. The stimuli were displayed on a Princeton graphics monochrome monitor under the control of an IBM PC AT. Subjects viewed the stereograms through a Keystone stereoscope (Model 50 ). The eye-to-screen distance for viewing through the stereoscope was $21.8 \mathrm{~cm}$.

Procedure. The subjects were told to position their hands on the keyboard as if they were typing, and to press the space bar on each trial as soon as they had a clear percept of the cross. Once the space bar was pressed, either a square or a circle appeared at the same location as the cross. If they saw a circle, they were not to respond to the target that followed. If they saw a square, they were to respond to the target that followed at the same location, pressing the J key with the right hand if they saw a vertical bar, or the F key with the left hand if they saw a horizontal bar. They were instructed to respond as quickly as possible, but also to be as accurate as possible. The subjects were also told that they might see other items lo- cated around the central target, but that they should ignore these other elements.

The subjects were shown six blocks of the displays, with each block containing eight replications of each display condition ( 7 disparity levels $\times 2$ noise types) and 16 catch trials (trials in which the circle precued the target), for a total of 128 trials per block. The 16 catch trials consisted of eight compatible and 8 incompatible noise trials with the disparity equal to the response target. The subjects were given a rest halfway through each block, and they were also allowed to rest following the completion of each block.

\section{Results and Discussion}

There were no significant differences in RT between vertical and horizontal shapes, either when they were presented as response targets $[t(15)=1.12]$, or when they were presented as noise elements $[t(15)=0.98]$. Additional analyses were therefore collapsed across this condition (see Figure 3). The mean RT for each subject for each condition was tabulated and analyzed in a two-way (noise condition $\times$ disparity) analysis of variance (ANOVA). The main effect for noise type was significant $[F(1,15)=6.01, p<.05]$. The mean RTs for the compatible and incompatible noise conditions were 556 and $563 \mathrm{msec}$, respectively. The main effect for disparity difference $[F(6,90)=1]$ and the interaction between the noise type and disparity value $[F(6,90)=1.32]$ were not significant $(p>.05)$. In order to reduce the degree of variability in the data, the scores for each subject were converted to standardized scores and analyzed in a second ANOVA. The main effect for the noise type was again significant $[F(1,15)=10.3, p<.01]$. The interaction between the noise type and the disparity value was also significant $[F(6,90)=2.25, p<.05]$. The main effect

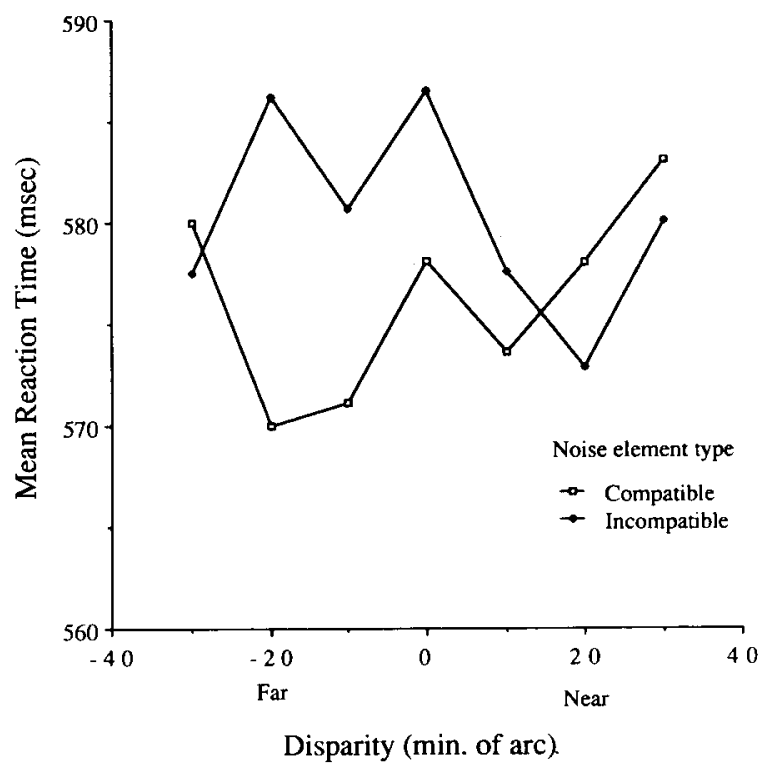

Figure 3. Interaction of the type of noise element with the disparity value of the noise element. The zero disparity value indicates that the response target and the noise element were located in the same disparity plane. 
for the difference in disparity $[F(6,90)=1.66]$ was not significant $(p>.05)$.

According to these results, the incompatible noise conditions, as compared with the compatible noise conditions, resulted in greater interference with the correct response. This result is consistent with results from other studies in which 2-D displays were used (B. A. Eriksen \& C. W. Eriksen, 1974; C. W. Eriksen \& Hoffman, 1973). As indicated in Figure 3, the interfering effect of incompatible noise was greatest when the noise elements were positioned farther away than the central target (the -20 ' disparity value). At the extreme depth conditions, there was an increase in RT for both compatible and incompatible noise elements. This is probably a result of the disparity value of the noise elements relative to the central target being close to the extreme limits of Panum's fusion area (Schumer \& Julesz, 1984). When the noise elements are positioned at these disparity values, the appearance of the elements might become diplopic (Duwaer, 1983). The present results indicate that noise elements at extreme disparity values interfere with the response to the central target, regardless of whether they are compatible or incompatible with the central target.

Another method of analyzing the data is to determine the differential interference of compatible and incompatible noise. This is obtained by subtracting the RTs for the compatible trials from the RTs for the incompatible trials. The results of this type of analysis are shown in Figure 4. A one-way ANOVA indicated that the effect of disparity on differential interference was significant $[F(6,90)=$ $2.73, p<.05]$. Post hoc comparisons (Tukey's HSD test) indicated significant differences between the $-20^{\prime}$ of arc disparity and the $20^{\prime}$ and $30^{\prime}$ of arc disparities.

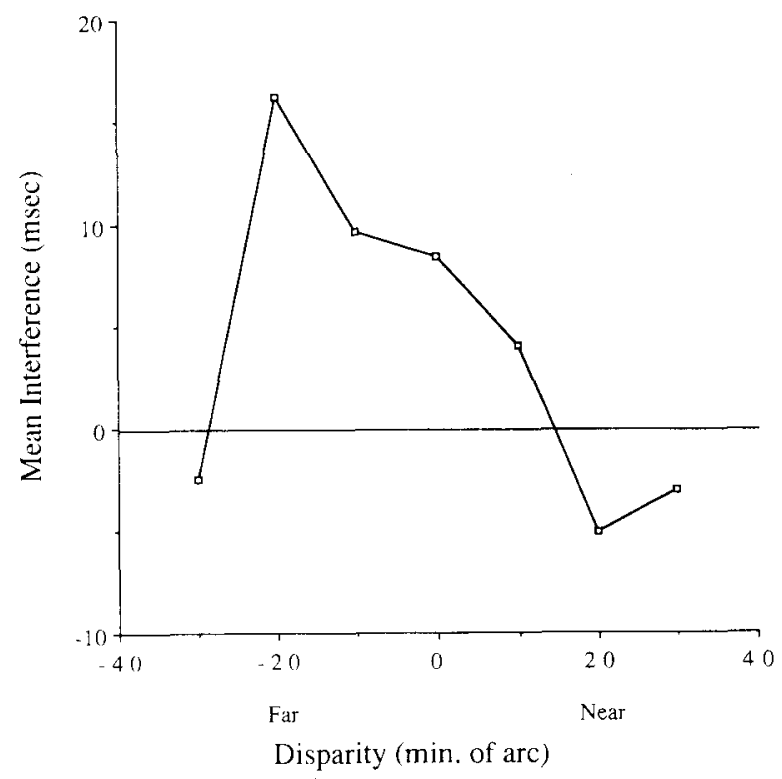

Figure 4. The differential interference of attention as a function of the disparity value of the noise elements.
Table 1

Percentage of Error Rates for Trials Requiring a Response

\begin{tabular}{cccccc}
\hline & \multicolumn{2}{c}{ Experiment 1} & & \multicolumn{2}{c}{ Experiment 2} \\
\cline { 2 - 3 } \cline { 5 - 6 } Disparity & Compatible & Incompatible & & Compatible & Incompatible \\
\hline-30 & 2.8 & 3.1 & & 2.8 & 3.5 \\
-20 & 2.6 & 3.0 & & 3.4 & 5.3 \\
-10 & 2.6 & 3.4 & & 2.4 & 3.4 \\
0 & 2.5 & 2.5 & & 3.0 & 3.6 \\
10 & 2.3 & 2.6 & & 3.1 & 5.2 \\
20 & 3.0 & 2.6 & & 2.6 & 4.6 \\
30 & 3.0 & 2.6 & & 3.1 & 3.2 \\
\hline
\end{tabular}

Note-Values for disparity are given in minutes of arc.

The mean increase in RT for the incompatible noise conditions at the position of greatest interference (the $-20^{\prime}$ disparity value) was 17 msec. When the noise elements were positioned at the same disparity value as the central target, there was a 10-msec increase in RT for the incompatible versus compatible noise. While this may not seem like a large effect, these results are similar to findings from other studies with 2-D displays (B. A. Eriksen \& C. W. Eriksen, 1974), when the separation between the central target and noise elements was $0.5^{\circ}$.

An additional analysis indicated that the differential interference of attention was greater for far (or uncrossed disparity) noise elements $(11 \mathrm{msec})$ than for near (or crossed disparity) noise elements $[-.4 \mathrm{msec} ; t(15)=$ $2.15, \mathrm{p}<.05]$. This asymmetrical effect for near and far distances supports the position that focused attention to items present in a 3-D display occurs within a viewercentered representation.

The error rates for trials requiring a response were also recorded (see Table 1) and analyzed in a two-way (noise type $\times$ disparity value) ANOVA. The main effects for noise type $[F(1,15)<1]$ and disparity value $[F(6,90)<$ 1] and the interaction between noise type and disparity value $[F(6,90)<1]$ were not significant. The mean percentages of errors for the compatible and incompatible noise elements were 2.68 and 2.82 , respectively. The error rates for catch trials (percentage of catch trials to which a subject responded) also showed no significant difference between compatible and incompatible conditions [3.12 vs. $3.03 ; t(15)=1.55$ ].

\section{EXPERIMENT 2}

In Experiment 1, the interfering effect of incompatible noise elements was found to vary as a function of binocular disparity. An asymmetry in the pattern of interference was found, indicating that elements that were positioned farther away produced greater interference than did elements that were closer. In Experiment 2, the same paradigm was used as in Experiment 1, except that the separation in visual angle between the central target and noise elements was decreased. This was accomplished by placing noise elements above and below the central target (see Figure 5). If focused attention occurs within a 

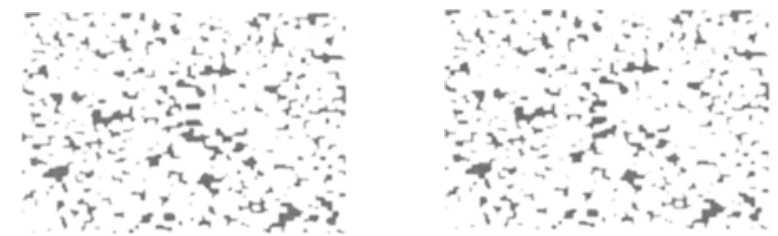

Figure 5. The response display used in Experiment 2.

limited area in the perceptual representation, then the effects of incompatible noise should be greater when the disparity value is identical to the value for the central target. This result would be consistent with results from other studies, in which the interfering effects of incompatible noise were found to increase when the visual angle between the central target and noise elements was decreased (B. A. Eriksen \& C. W. Eriksen, 1974).

Although fewer noise elements were present in the response display than in the displays used in Experiment 1, the spatial separation between the noise elements and central target was reduced relative to those conditions. In previous studies, greater interference of noise elements has been found when the elements were located within $1^{\circ}$ of visual angle. Indeed, noise elements that were $0.06^{\circ}$ from the central target resulted in increased reaction times of up to $80 \mathrm{msec}$ (B. A. Eriksen \& C. W. Eriksen, 1974). Therefore, it was expected that the decreased spacing between the central target and noise elements would result in greater interference for incompatible noise conditions.

\section{Method}

Subjects. The subjects were 10 University of Illinois undergraduates who were paid for their participation in the study. All subjects had normal or corrected-to-normal vision. One subject was not run in the study, because of failure to show sensitivity to binocular disparity information on a Randot stereotest.

Design. Two independent variables were examined: type of noise element (compatible or incompatible) and difference in disparity between the central target and noise elements $\left(-30^{\prime},-20^{\prime},-10^{\prime}\right.$, $0^{\prime}, 10^{\prime}, 20^{\prime}$, or $30^{\prime}$ of arc).

Stimuli. The displays were similar to those used in Experiment 1, with the following exception. Only two noise elements were presented and were located above and below the central target. The edge-to-edge separation between the noise elements and central target was $0.13^{\circ}$.

Apparatus and Procedure. The apparatus and procedure were the same as those used in Experiment 1.

\section{Results and Discussion}

Mean RT did not differ between vertical and horizontal shapes, either when they were presented as response targets $[t(8)=0.92]$ or when they were presented as noise elements $[t(8)=1.13]$. Additional analyses were therefore collapsed across this condition (see Figure 6). The mean RT for each subject for each condition was tabulated and analyzed in a two-way (noise type $\times$ disparity value) ANOVA. The main effect for noise type was significant $[F(1,8)=5.84, p<.05]$. The mean RTs for the compatible and incompatible noise conditions were 554 and $564 \mathrm{msec}$, respectively. Thus, as in Experiment 1, the incompatible noise conditions resulted in greater interference with the correct response, as compared with the compatible noise conditions. The main effect for the difference in disparity $[F(6,48)<1]$ and the interaction between noise type and disparity value $[F(6,48)=1.92]$ were not significant $(p>.05)$. In order to reduce the degree of variability in the data, the scores for each subject were converted to standardized scores and analyzed in a second ANOVA. The main effect for the noise type was again significant $[F(1,8)=7.61, p<.01]$. The interaction between the noise type and the disparity value was also significant $[F(6,48)=2.74, p<.05]$. The main effect for the difference in disparity was not significant $[F(6,48)<1]$.

A one-way ANOVA found that the effect of disparity on differential interference was significant $[F(6,48)=$ $3.08, p<.05]$. Post hoc comparisons (Tukey's $H S D$ test) indicated significant differences between the $0^{\prime}$ and the $20^{\prime}$ and $30^{\prime}$ of arc disparities. As indicated in Figure 6, the interfering effect of incompatible noise was greatest when the noise elements were at the same disparity value (and thus the same depth plane) as the central target. Indeed, the mean increase in RT was $21 \mathrm{msec}$, which is similar to the $28-\mathrm{msec}$ result obtained by extrapolating between the $0.06^{\circ}$ and $0.5^{\circ}$ conditions of B. A. Eriksen and C. W. Eriksen (1974; see their Figure 1).

An additional analysis compared the effects of far (uncrossed disparity) and near (crossed disparity) positions of the noise elements on the differential interference of attention. Incompatible noise elements that were positioned farther away from the central target produced

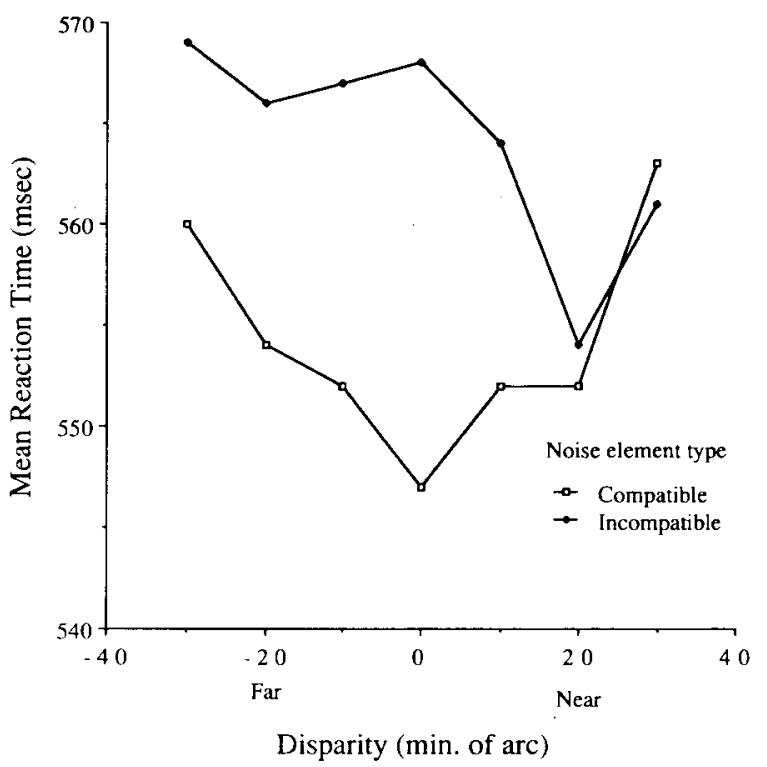

Figure 6. Interaction of the type of noise element with the disparity value of the noise element. The zero disparity value indicates that the response target and the noise element were located at the same depth plane. 


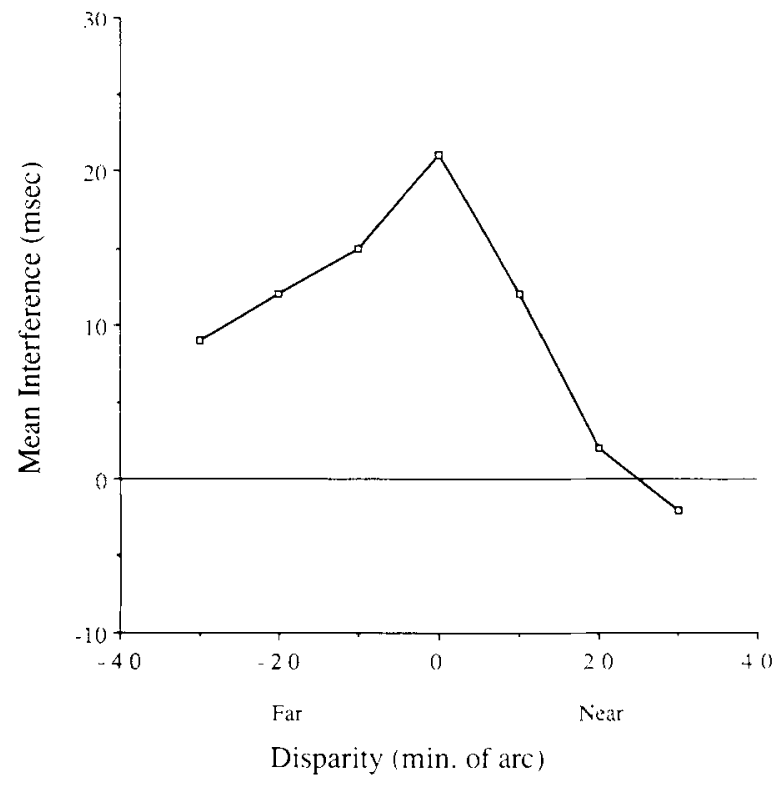

Figure 7. The differential interference of attention as a function of the disparity value of the noise elements.

greater interference $[t(8)=2.35, p<.05]$ (see Figure 7). The mean interferences for near and far positions were 4 and $12 \mathrm{msec}$, respectively. The asymmetrical effect for near and far positions provides further evidence that focused attention from 3-D displays occurred within a viewer-centered representation.

The error rates for trials requiring a response were also recorded (see Table 1) and analyzed in a two-way (noise type $x$ disparity value) ANOVA. The main effects for noise type $[F(1,8)=3.5]$ and disparity value $[F(6,48)$ $=1.43]$ and the interaction between noise type and disparity value $[F(6,48)<1]$ were not significant. The mean percentage of errors for the compatible and incompatible noise elements were 3.31 and 4.11 , respectively. Significantly more catch trial errors occurred for compatible than for incompatible conditions $[3.72$ vs. $1.54 ; t(8)=2.96$, $p<.01]$.

\section{GENERAL DISCUSSION}

The present results support the hypothesis that focused attention is restricted to a limited region in 3-D space. According to the results from Experiments 1 and 2, the effects of incompatible noise elements varied with increased distance in 3-D from the central target. The notion of a gradient of attention or processing resources across visual space is consistent with this result, as well as with the results from other studies in which focused attention in 2-D space has been investigated. The primary difference in the present study is that the gradient was surrounding a position along the line of sight as a function of distance defined by binocular disparity.

The asymmetrical effect of incompatible noise elements as a function of distance (i.e., greater effect of far ele- ments than of near elements) is consistent with the concept that the allocation of processing resources from 3-D information is based on a viewer-centered representation. There are several reasons why this should be the case. First, it seems unlikely that our capacity for attending to relevant information within a scene would be independent of information regarding its position relative to the viewer. Second, research from several different domains has provided evidence that our perceived visual representation involves viewer-centered information. Research in visual imagery (Roth \& Kossyln, 1988) and memory recall (Jolicoeur \& Kosslyn, 1983) has yielded evidence that our internal visual representation can be viewer-centered in nature. In addition, recent evidence in perceptual studies (Braunstein, Tittle, \& Myers, 1988) suggests that we recover a viewer-centered representation even when we are provided only with object-centered information.

The present results are also consistent with the concept that attention operates as a spotlight within the visual field. According to this view, attention consists of the allocation of resources to a specific location in space. The present results extend the spotlight analogy to a 3-D representation of the world, and suggest that the focus of attention may be described as a solid visual angle originating from the viewer's position in the representation with asymmetrical depth of focus (see Figure 8). Far noise elements (i.e., those positioned at a greater distance than the central focus of attention) may lie in the beam of attention, because the size of focused attention varied with distance. This would provide a partial explanation of the asymmetrical effects of incompatible noise as a function of the level of crossed versus uncrossed disparity. However, this cannot by itself explain the asymmetrical interference, because noise elements in both experiments were positioned at a constant visual angle. If focused attention in depth were limited to a conical region, then equal interference should have been found for several depth positions around the position of the attended target.

The results of the present experiments suggest that the 2-D size of attention varied with distance. An additional issue that could account for the changing size of attention is that focused attention varied with distance much as the depth of focus of a camera is asymmetrical along the depth axis. According to this analogy, the depth of focus (and thus the 2-D size of attention) was narrower for near targets as compared with more distant targets. If this metaphor is appropriate, then variations in the absolute distance of the response target should result in changes in the size of focused attention, much as the depth of focus varies according to the distance between a camera and the object in focus. The effect of variations in absolute distance between the observer and an attended object on the size of focused attention in 3-D space would be an important issue to address in future research.

There are two alternative explanations that could account for the asymmetrical effect of interference produced by noise elements located at near and far depth positions. The first possibility is that noise elements positioned far- 


\section{Horizontal Axis}

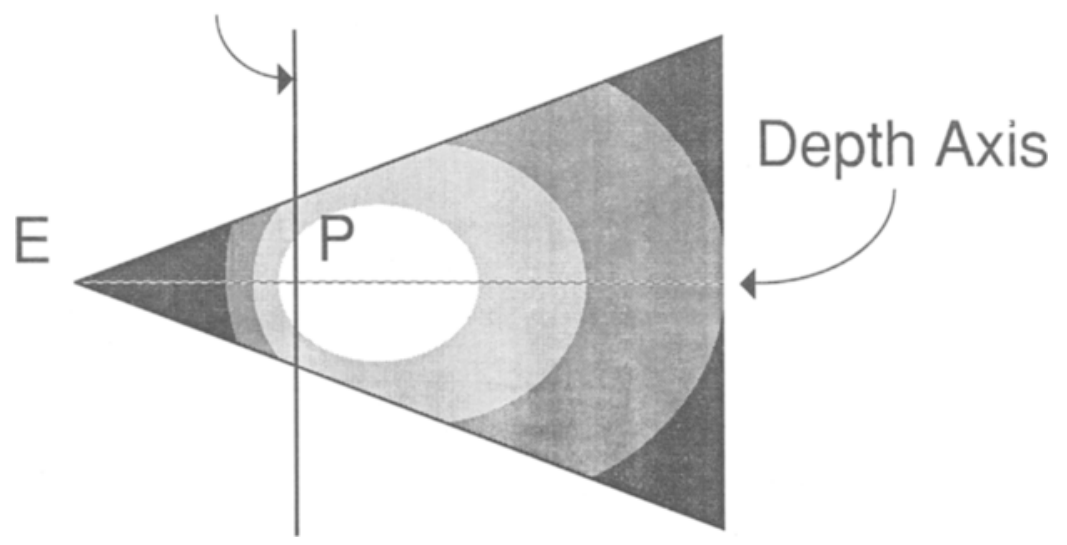

Figure 8. Pictorial representation of a top view of focused attention within a viewercentered representation. $E$ represents the eye position of the viewer. $P$ represents the attended position within the representation. Variations in shading represent varying amounts of allocated processing resources for focused attention.

ther away may have been perceived as larger than noise elements positioned at closer distances. This could have occurred because the visual angle of the elements was constant but the position in depth was varied. Larger noise elements have been shown to influence same versus different RTs (Watson, 1981) and to produce greater interference than smaller elements do in a response compatibility paradigm (C. W. Eriksen \& Schultz, 1979). A second possibility is that the noise elements positioned farther away were perceived as closer to the target than nearer noise elements were, because of the compression of perceived visual space along the depth axis (Indow, 1982). According to this hypothesis, greater interference would have been produced because the farther noise elements appeared closer to the response target. An important topic for future research would be to determine the role of perceived size and the compression of visual space on the interfering effects of noise elements that vary in depth.

The present results contrast with results of previous research on attention in 3-D space. Downing and Pinker (1985) found that the cost of attention increased for more eccentric regions in the visual field. When subjects were required to detect targets within a small area of the central field (less than $2^{\circ}$ ), there was little cost in detecting targets at different depth planes. The present results indicate that the interfering effects of attending to incompatible noise can be considerable. Indeed, in Experiment 1 , the interference was $17 \mathrm{msec}$ for noise elements that had a $-20^{\prime}$ of arc disparity difference relative to the central target. The visual angle separation between the central stimulus and noise elements was approximately $1^{\circ}$. Of course there are several differences between the present study and that of Downing and Pinker. Their subjects had a variety of cues available for determining distance (accommodation, linear perspective, and texture), whereas the only cue in the present study was binocular disparity. In addition, Downing and Pinker were con- cerned with the effects of depth on responses to targets up to $16^{\circ}$ from the point of fixation, whereas in the present study, responses were made to targets located at the point of fixation.

The effects of variations in the position of noise elements along the depth axis on focused attention have been considered in the present study. In previous research, the effects of variations in the horizontal and vertical position of noise elements have been considered. In order to derive a more complete measure of the region of focused attention in the 3-D representation, future research is needed, in which the vertical and horizontal separations, together with the depth separation, can be varied between the noise elements and the central target. This would allow a mathematical calculation of an isocontour map of interference relative to the attended position in 3-D space.

\section{REFERENCES}

Braunstein, M. L. (1988). The empirical study of structure from motion. In W. N. Martin \& J. K. Aggarwal (Eds.), Motion understanding: Robot and human vision (pp. 101-142). Boston: Kluwer Academic Publishers.

Braunstein, M. L., Tittle, J. S. \& Myers, T. S. (1988). Size constancy in orthographic structure-from-motion projections. Investigative Ophthalmology \& Visual Science, 29, 249.

Downing, C., PINkER, S. (1985). The spatial structure of visual attention. In M. Posner \& O. Martin (Eds.), Attention and performance $X I$ (pp. 171-187). Hillsdale, NJ: Erlbaum.

DUNCAN, J. (1984). Selective attention and the organization of visual information. Journal of Experimental Psychology: General, 113, $501-517$

Duwaer, A. L. (1983). Patent stereopsis with diplopia in random-dot stereograms. Perception \& Psychophysics, 33, 443-454.

ErIKSEN, B. A., \& ErIKSEN, C. W. (1974). Effects of noise letters upon the identification of a target letter in a nonsearch task. Perception \& Psychophysics, 16, 143-149.

Eriksen, C. W., \& Hoffman, J. E. (1973). The extent of processing of noise elements during selective encoding from visual displays. Perception \& Psychophysics, 14, 155-160.

ERKSEN, C. W., \& SchulTz, D. W. (1979). Information processing 
in visual search: A continuous flow conception and experimental results. Perception \& Psychophysics, 25, 249-263.

ERIKSEN, C. W., \& ST. JAMES, J. D. (1986). Visual attention within and around the field of focal attention: A zoom lens model. Perception \& Psychophysics, 40, 225-240.

ERIKSEN, C. W. \& YEH, Y. (1985). Allocation of attention in the visual field. Journal of Experimental Psychology: Human Perception \& Performance, 11, 583-597.

Gawryszewski, L. D. G., Riggio, L., Rizzolatti, G., \& Umiltà, C. (1987). Movements of attention in the three dimensions and the meaning of "neutral" cues. Neuropsychologia, 25, 19-29.

Hoffman, J. E., \& Nelson, B. (1981). Spatial selectivity in visual search. Perception \& Psychophysics, 30, 283-290.

INDOw, T. (1982). An approach to geometry of visual space with no a priori mapping functions: Multidimensional mapping according to Riemannian metrics. Joumal of Mathematical Psychology, 26, 204-236.

Jolicoevr, P., Kosslyn, S. M. (1983). Coordinate systems in the long-term memory representation of three-dimensional shapes. Cognitive Psychology, 15, 301-345.

JuLEsz, B. (1971). Foundations of cyclopean perception. Chicago: University of Chicago Press.

LABERGE, D. (1983). Spatial extent of attention to letters in words. Journal of Experimental Psychology: Human Perception \& Performance, 9, 371-379.

LABERGE, D., Brown, V. (1986). Variations in size of the visual field in which targets are presented: An attentional range effect. Perception \& Psychophysics, 40, 188-200.
LABERGE, D., \& Brown, V. (1989). Theory of attentional operations in shape identification. Psychological Review, 96, 101-124.

MARR, D. (1982). Vision. San Francisco: W. H. Freeman.

MarR, D., \& Nishihara, H. K. (1978). Representation and recognition of the spatial organization of three-dimensional shapes. Proceedings of the Royal Society Series B, 200, 269-294.

Nakayama, K., \& Silverman, G. (1986). Serial and parallel processing of visual feature conjunctions. Nature, 320, 264-265.

Posner, M. I., Nissen, M. J., \& OGden, W. C. (1978). Attended and unattended processing modes: The role of set for spatial location. In H. L. Pick \& E. J. Saltzman (Eds.), Modes of perceiving and processing information (pp. 137-157). Hillsdale, NJ: Erlbaum.

RASHBASS, C., \& WESTHEIMER, G. (1961). Independence of conjugate and disjunctive eye movements. Joumal of Physiology, 159, 361-364.

Roth, J. D., KossLYN, S. M. (1988). Construction of the third dimension in mental imagery. Cognitive Psychology, 20, 344-361.

SCHUMER, R., \& Julesz, B. (1984). Binocular disparity modulation sensitivity to disparities offset from the plane of fixation. Vision Research, 24, 533-542.

Watson, H. D. (1981). The effects of objective and perceived size properties on visual form matching. Journal of Experimental Psychology: General, 110, 547-567.

Westheimer, G., Mitchell, D. E. (1969). The sensory stimulus for disjunctive eye movements. Vision Research, 9, 749-755.

(Manuscript received November 7, 1988; revision accepted for publication September 1, 1989.) 